\title{
COMBIEN DE PERSONNES ONT DÉVELOPPÉ DES SYMPTÔMES OU CONTRACTÉ LA COVID-19 AU QUÉBEC? Une étude exploratoire
}

DAVID BOISCLAIR ROXANE BORGÈS DA SILVA VINCENT BOUCHER NATHALIE DE MARCELLIS-WARIN PIERRE-CARL MICHAUD INGRID PEIGNIER 
Centre interuniversitaire de recherche en analyse des organisations

Les cahiers de la série scientifique visent à rendre accessibles les résultats des recherches effectuées par des chercheurs membres du CIRANO afin de susciter échanges et commentaires. Ces cahiers sont rédigés dans le style des publications scientifiques et $n^{\prime}$ engagent que leurs auteurs.

The purpose of the Working Papers is to disseminate the results of research conducted by CIRANO research members in order to solicit exchanges and comments. These reports are written in the style of scientific publications. The ideas and opinions expressed in these documents are solely those of the authors.

Le CIRANO est un organisme sans but lucratif constitué en vertu de la Loi des compagnies du Québec. Le financement de son infrastructure et de ses activités de recherche provient des cotisations de ses organisations-membres, d'une subvention d'infrastructure du gouvernement du Québec, de même que des subventions et mandats obtenus par ses équipes de recherche.

CIRANO is a private non-profit organization incorporated under the Quebec Companies Act. Its infrastructure and research activities are funded through fees paid by member organizations, an infrastructure grant from the government of Quebec, and grants and research mandates obtained by its research teams.

Les partenaires du CIRANO - CIRANO Partners

Partenaires corporatifs - Corporate Partners

Autorité des marchés financiers

Banque de développement du Canada

Banque du Canada

Banque nationale du Canada

Bell Canada

BMO Groupe financier

Caisse de dépôt et placement du Québec

Énergir

Hydro-Québec

Innovation, Sciences et Développement économique Canada

Intact Corporation Financière

Investissements PSP

Manuvie Canada

Ministère de l'Économie, de la Science et de l'Innovation

Ministère des finances du Québec

Mouvement Desjardins

Power Corporation du Canada

Rio Tinto

Ville de Montréal

Partenaires universitaires - Academic Partners

École de technologie supérieure

École nationale d'administration publique

HEC Montréal

Institut national de la recherche scientifique

Polytechnique Montréal

Université Concordia

Université de Montréal

Université de Sherbrooke

Université du Québec

Université du Québec à Montréal

Université Laval

Université McGill

Le CIRANO collabore avec de nombreux centres et chaires de recherche universitaires dont on peut consulter la liste sur son site web. CIRANO collaborates with many centers and university research chairs; list available on its website.

(C) Janvier 2022. David Boisclair, Roxane Borgès Da Silva, Vincent Boucher, Nathalie De Marcellis-Warin, Pierre-Carl Michaud et Ingrid Peignier. Tous droits réservés. All rights reserved. Reproduction partielle permise avec citation du document source, incluant la notice (c). Short sections may be quoted without explicit permission, if full credit, including (C) notice, is given to the source.

Les idées et les opinions émises dans cette publication sont sous l'unique responsabilité des auteurs et ne représentent pas nécessairement les positions du CIRANO ou de ses partenaires. The observations and viewpoints expressed in this publication are the sole responsibility of the authors; they do not necessarily represent the positions of CIRANO or its partners. 


\title{
COMBIEN DE PERSONNES ONT DÉVELOPPÉ DES SYMPTÔMES OU CONTRACTÉ LA COVID-19 AU QUÉBEC? Une étude exploratoire
}

\author{
David Boisclair*, Roxane Borgès Da Silva ${ }^{\dagger}$, Vincent Boucher ${ }^{\ddagger}$, Nathalie De \\ Marcellis-Warin $^{\S}$, Pierre-Carl Michaud ${ }^{* *}$ et Ingrid Peignier ${ }^{\dagger \dagger}$
}

\begin{abstract}
Résumé
Nous estimons l'incidence de la COVID-19, soit le nombre de personnes adultes ayant reçu un résultat positif à un test, rapide ou PCR, ou s'étant auto-diagnostiquées comme ayant eu la COVID-19 sur la base des symptômes, sur une période de sept jours se terminant entre le 13 et le 18 janvier 2022. Nous présentons une estimation par échantillonnage direct sur la population adulte ainsi qu'une estimation par échantillonnage indirect basée sur la méthode de l'amplificateur par réseau (network scale up method).

Nos résultats suggèrent que durant cette période 261863 personnes ont reçu un résultat positif à un test, ce qui est semblable aux estimations par la méthode de l'amplificateur par réseau (variant de 231967 à 251706 selon la méthode). Sur une base journalière, ces chiffres indiquent qu'il y avait en moyenne 33000 à 40000 tests positifs par jour au Québec durant la période. De plus, 146567 personnes rapportent un auto-diagnostic positif, ce qui une fois ajouté aux personnes ayant reçu un résultat de test positif donne une estimation de 407430 cas probables de COVID-19 au sein de la population adulte (58 144 en moyenne par jour). Comparativement, les données de I'INSPQ rapportent 48815 cas positifs sur la base de tests PCR au Québec durant cette même période de sept jours (6 973 en moyenne par jour). Peu importe la méthode et l'estimateur utilisé (en excluant les auto-diagnostics), nous obtenons une incidence qui est environ cinq fois plus élevée que le chiffre officiel. L'enquête sera répétée sur quatre semaines consécutives dans un objectif de suivre l'évolution.
\end{abstract}

\footnotetext{
*HEC Montréal

† Université de Montréal et CIRANO

‡ Université Laval

$\S$ Polytechnique Montréal et CIRANO

${ }^{* *}$ HEC Montréal et CIRANO

†† CIRANO
}

Abstract

We estimate the incidence of COVID-19, which is the number of adult persons who tested positive for COVID-19, either rapid or PCR, or self-diagnosed as having COVID-19 on the basis of 
symptoms, over a 7-day period ending January 13-18, 2022. We present a direct sampling estimate on the adult population as well as an indirect sampling estimate based on the network scale-up method.

Our results suggest that during this period 261,863 people tested positive, which is similar to the network scale-up estimates (ranging from 231,967 to 251,706 depending on the method). On a daily basis, these figures indicate that there were an average of 35,000 to 40,000 positive tests per day in Quebec during the period. In addition, 146,567 people report a positive selfdiagnosis, which when added to those with a positive test result gives an estimate of 407,430 probable cases of COVID-19 in the adult population (58,144 on average per day). In comparison, INSPQ data report 48,815 positive cases based on PCR tests in Quebec during the same sevenday period (6,973 on average per day). Regardless of the method and estimator used (excluding self-diagnosis), we obtain an incidence that is about five times higher than the official figure. The survey will be repeated over 4 consecutive weeks in order to follow the evolution.

Mots-clés/Keywords : COVID-19, Québec , amplificateur par réseau, incidence / COVID-19, Quebec, Network Scale Up Method (NSUM), incidence

Codes JEL/JEL Codes : C83 et I18

\section{Pour citer ce document / To quote this document}

Boisclair, D., Borgès Da Silva, R., Boucher, V., De Marcellis-Warin, N., Michaud, P-C et Peignier, I. (2022). Combien de personnes ont développé des symptômes ou contracté la Covid-19 au Québec? Une étude exploratoire (2022s-03, CIRANO).

https://doi.org/10.54932/KWYT2364 


\title{
Combien de personnes ont développé des symptômes ou contracté la COVID-19 au Québec? Une étude exploratoire *
}

\author{
David Boisclair Roxane Borgès Da Silva Vincent Boucher \\ Nathalie de Marcellis-Warin Pierre-Carl Michaud Ingrid Peignier
}

21 janvier 2022

\section{Résumé}

Nous estimons l'incidence de la COVID-19, soit le nombre de personnes adultes ayant reçu un résultat positif à un test, rapide ou $P C R$, ou s'étant auto-diagnostiquées comme ayant eu la COVID-19 sur la base des symptômes, sur une période de sept jours se terminant entre le 13 et le 18 janvier 2022. Nous présentons une estimation par échantillonnage direct sur la population adulte ainsi qu'une estimation par échantillonnage indirect basée sur la méthode de l'amplificateur par réseau (network scale up method). Nos résultats suggèrent que durant cette période 261863 personnes ont reçu un résultat positif à un test, ce qui est semblable aux estimations avec

*Boisclair: HEC Montréal, Borgès Da Silva: Université de Montréal et CIRANO, Boucher: Université Laval, De Marcellis-Warin: Polytechnique Montréal et CIRANO, Michaud: HEC Montréal et CIRANO, Peignier: CIRANO. Les auteurs déclarent n'avoir aucun conflit d'intérêt dans le cadre de ce projet. Les données de cette étude proviennent d'une enquête développée par le CIRANO et administrée par la firme Léger. Les données ainsi que les scripts ayant produit les résultats de cette note sont disponibles sur le site https://www.github. $\mathrm{com} / \mathrm{pcmichaud/EnqueteCovid.git.}$ la méthode de l'amplificateur par réseau (variant de 231967 à 251706 selon la méthode). Sur une base journalière, ces chiffres indiquent qu'il y avait en moyenne 33000 à 38000 tests positifs par jour au Québec durant la période. De plus, 146567 personnes rapportent un auto-diagnostic positif, ce qui une fois ajouté aux personnes ayant reçu un résultat de test positif, donne une estimation de 407430 cas probables de COVID19 au sein de la population adulte (58 144 en moyenne par jour). Comparativement, les données de l'INSPQ rapportent 48815 cas positifs sur la base de tests PCR au Québec durant cette même période de sept jours (6973 en moyenne par jour). Peu importe la méthode et l'estimateur utilisé (en excluant les auto-diagnostics), nous obtenons une incidence qui est environ cinq fois plus élevée que le chiffre officiel. L'enquête sera répétée sur quatre semaines consécutives dans un objectif de suivre l'évolution. 


\section{Contexte}

La $5^{\mathrm{e}}$ vague de la COVID-19 est caractérisée par une montée en flèche des cas de COVID-19. Le nombre de cas quotidien est passé de 1296 le $1^{\text {er }}$ décembre 2021 à 16547 le 29 décembre, comme le montre la Figure 1. La capacité à réaliser des tests PCR en centre de dépistage a rapidement été dépassée. De plus, la montée fulgurante du taux de positivité, atteignant plus de $30 \%$ autour du Nouvel An, ${ }^{1}$ indique probablement que seulement les cas les plus graves étaient testés et qu'un grand nombre ne l'était plus. La forte demande pour les tests PCR et les délais d'attente pour obtenir les résultats ont mené à une directive du ministère de la Santé et des Services sociaux du Québec, en date du 4 janvier 2022, de réserver ces tests seulement aux clientèles prioritaires et aux travailleurs essentiels. ${ }^{2}$ À partir de cette date, la population générale a donc uniquement pu se baser sur les tests rapides antigéniques pour connaître son statut d'infection au virus. En plus des délais dans le déploiement de la distribution des tests rapides et d'une offre probablement insuffisante, les résultats des tests rapides ne sont pas répertoriés publiquement ce qui complique la mesure de l'incidence des cas de COVID-19 au Québec.

Cette absence de mesure à ce stade dans cette $5^{\mathrm{e}}$ vague est problématique pour deux raisons : 1) le nombre de nouveaux cas est un indicateur avancé des hospitalisations à venir; et 2) la mesure du taux d'hospitalisation parmi ceux ayant la COVID-19 permet de juger de la sévérité du variant Omicron, et donc des mesures qui doivent

1. Données INSPQ : https://www.inspq.qc.ca/ covid-19/donnees.

2. La directive du MSSS peut être consultée à https://www.msss.gouv.qc.ca/ministere/ salle-de-presse/communique-3371/. être mises en place pour réduire sa propagation afin de minimiser les risques d'hospitalisations et les décès.

Dans cette note, nous rapportons les résultats d'une étude exploratoire menée par le CIRANO visant à mesurer l'incidence de la COVID-19 par enquête. Cette note, en plus de décrire l'originalité de la méthode utilisée, présente quelques premiers résultats intéressants issus d'une première collecte de données ayant eu lieu du 13 au 18 janvier 2022. Quatre autres collectes sont prévues et seront échelonnées sur les quatre semaines suivantes. Bien que la prévalence actuelle permette de procéder par échantillonnage direct, nous anticipons que la taille d'échantillon requise, pour mesurer l'incidence des cas avec une certaine précision, augmentera nettement avec la baisse des cas anticipée au cours des prochaines semaines. Nous avons donc aussi recours à une technique d'estimation par échantillonnage indirect, dite de l'amplificateur par réseau (APR; en anglais Network Scale Up Method, ou NSUM). L'estimation par échantillonnage direct nous permet de valider la qualité des estimations obtenues par cette méthodologie.

\section{Méthode}

Nous présentons ici brièvement l'APR. Tel que discuté, la taille d'une petite sous-population est difficile à estimer par échantillonnage direct sur une taille d'échantillon raisonnable. Un échantillon de 1000 répondants risque très souvent de n'avoir aucun répondant provenant d'une petite sous-population (1 sur 100000 par exemple). Plusieurs techniques existent pour palier ce problème. L'APR consiste à exploiter les réseaux des répondants afin d'obtenir une estimation plus précise de la taille d'une sous-population dans 

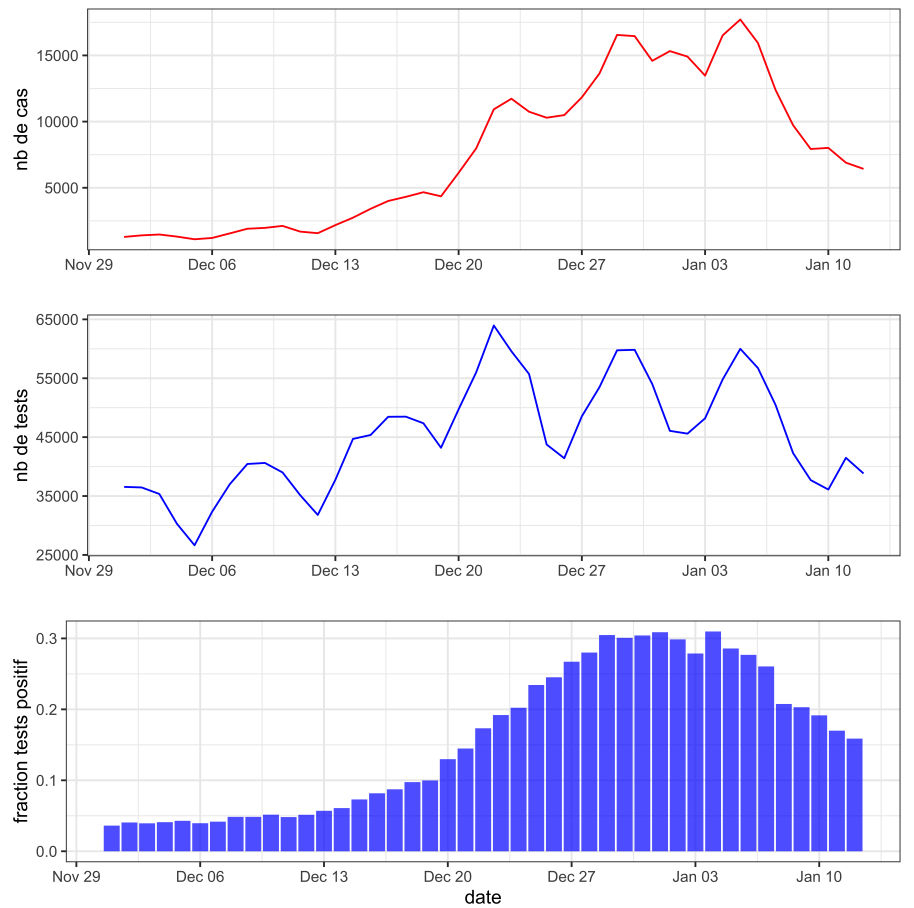

Figure 1 - Évolution des cas, des tests PCR et de la fraction de tests positifs lors de la $5^{\text {e }}$ vague au Québec. Données de l'INSPQ colligées le 13 janvier 2022, basées sur les tests PCR effectués en centre de dépistage au Québec.

un petit échantillon. Cette technique a d'abord été utilisée afin d'estimer le nombre de décès dus à un tremblement de terre au Mexique (Bernard et al., 1991). Plusieurs raffinements ont été proposés et sont recensés par Laga et al. (2021). L'avantage de cette méthode est qu'elle est aussi particulièrement adaptée pour capter une sous-population volatile (Rolland-Guillard et al., 2020), c'est-à-dire qui entre et sort de la population très rapidement, comme c'est le cas pour la COVID-19 qui est éphémère dans le temps.

Supposons une population de taille $N$ et des sous-populations $k=1, \ldots K$ de taille $N_{k}$. Supposons que pour $L$ sous-populations, nous connais- sions la taille exacte de la sous-population, mais que la taille d'une sous-population, $N_{u}$ nous soit inconnue.

Prenons un échantillon aléatoire de taille $n$ de la population générale de taille $N$. Une donnée relationnelle agrégée (DRA) est la réponse à une question sur le nombre de connaissances d'un répondant dans une sous-population donnée : «Combien de personnes de la sous-population $k$ connaissez-vous? ». Notons la réponse à une question DRA du répondant $i$ sur la population $k$ par $y_{i k}$. Nous pouvons poser une question de type DRA pour chaque sous-population $k=1, \ldots, L$ ainsi que pour la sous-population $u$ de telle sorte que les réponses sont données par 
le vecteur $y_{i}=\left(y_{i 1}, \ldots, y_{i L}, y_{i u}\right)$.

L'avantage de l'utilisation des DRA est que nous pouvons obtenir un échantillonnage (indirect) d'une petite sous-population, même avec un échantillon de faible taille. Par exemple, un échantillon de 1000 individus connaissant chacun 100 autres individus est équivalent à un échantillonnage direct de 100000 individus. ${ }^{3}$ « Le réseau de connaissances des répondants devient alors un échantillon artificiel $\gg$ (Rolland-Guillard et al., 2020).

Ce type d'analyse dépend par contre d'une hypothèse importante :

$$
\mathbb{E} y_{i k}=\alpha_{i} N_{k},
$$

où $\mathbb{E} y_{i k}$ est l'espérance de la variable relationnelle agrégée $y_{i k}$ et $\alpha_{i}$ est une variable latente inobservée.

L'hypothèse (1) présentée ici est moins restrictive que celles habituellement utilisées dans la littérature mais est suffisante à la convergence des deux principaux estimateurs, soit celui de Killworth et al. (1998) ainsi que celui de Habecker et al. (2015), formellement définis respectivement comme suit :

$$
N_{u}^{K}=\mathbb{E} y_{i u} \frac{\sum_{k} N_{k}}{\sum_{k} \mathbb{E} y_{i k}}
$$

et

$$
N_{u}^{H}=\mathbb{E} y_{i u} \frac{1}{K} \sum_{k} \frac{N_{k}}{\mathbb{E} y_{i k}} .
$$

La condition (1), sous-jacente à la validité de ces deux estimateurs, implique que le réseau de connaissances des individus est, en moyenne, représentatif de la population.

L'intuition derrière ces estimateurs est simple. Ayant un échantillon tiré de manière aléatoire

3. Pour les fins de l'exemple, nous supposons que les connaissances des individus sont mutuellement exclusives. dans la population, si le réseau de connaissances des individus est représentatif de la population en général (équation (1)), alors les DRA permettent d'estimer la taille des souspopulations. Cette conclusion est valide peu importe le nombre de connaissances que les individus ont. Ainsi, plutôt que d'avoir seulement $n$ réponses sur lesquelles baser notre inférence sur $N_{u}$, l'utilisation des réseaux nous permet d'exploiter l'inférence que chacun des répondants fait sur son propre réseau afin d'estimer de manière plus précise la taille de la sous-population inconnue.

Par contre, puisque l'échantillonnage est indirect et que nous ne disposons que de peu d'information sur le réseau de connaissances des individus, cette méthode d'estimation peut souffrir de certains biais potentiels, bien connus dans la littérature. ${ }^{4}$ En particulier ici, l'APR sous-estime potentiellement le nombre de cas, puisque les individus ne sont pas nécessairement au fait de l'ensemble des cas positifs au sein de leur réseau de connaissances au cours de la dernière semaine (effet de transmission; voir p.ex. Killworth et al. 2003), d'autant plus qu'il existe encore une certaine stigmatisation des personnes ayant eu la COVID-19. Finalement un autre biais peut venir du fait que la probabilité que les répondants connaissent des personnes ayant eu la COVID19 n'est pas uniforme et pourrait dépendre de certaines caractéristiques sociodémographiques (par exemple, une personne travaillant dans le secteur de la santé connaît certainement plus de personnes ayant eu la COVID-19).

Quoi qu'il en soit, puisque que la prévalence actuelle nous permet de procéder à la fois par échantillonnage direct et indirect, nous pouvons évaluer la performance des estimateurs par APR

4. Voir Laga et al. (2021) pour une discussion détaillée. 
et les calibrer. Ce calibrage sera fort utile lors des collectes subséquentes de l'enquête, alors que le nombre de cas sera potentiellement plus faible.

\section{Enquête}

Cet article présente les réponses d'un échantillon représentatif de la population du Québec qui a été interrogé par Internet du 13 au 18 janvier 2022. Les répondants, tous âgés de plus de 18 ans, pouvaient s'exprimer en français ou en anglais. Au total, 3000 personnes sélectionnées selon la méthode des quotas et des strates ont répondu au questionnaire. La durée moyenne pour répondre au questionnaire de l'enquête a été de 6 minutes 14 secondes avec une médiane de 4 minutes et 45 secondes. L'enquête a été réalisée avec le panel en ligne Léger Opinion (LEO). Les répondants ont été sélectionnés de façon aléatoire dans le panel.

À l'aide des statistiques du Recensement de 2016 de Statistique Canada, les résultats ont été pondérés par la firme de sondage selon l'âge, le sexe, la langue maternelle, le niveau de scolarité, la présence d'enfants ou non et la région afin de rendre l'échantillon représentatif de l'ensemble de la population adulte du Québec.

Le questionnaire comporte une série de dix questions plus une dizaine de questions sociodémographiques. ${ }^{5}$ Le questionnaire vise dans un premier temps à poser aux répondants des questions sur leur propre expérience avec la COVID19 au cours des sept derniers jours. Par la suite une série de questions sont posées visant le réseau de connaissances de chaque répondant. La section se termine par une question sur la vaccination. Finalement, des questions sur le profil

5. Une copie du questionnaire se trouve en annexe de cette note. sociodémographique des répondants sont posées.

La question permettant d'estimer l'incidence de la COVID-19 au cours des sept derniers jours à partir de la méthode d'échantillonnage direct est la suivante :

«Dans les sept derniers jours incluant aujourd'hui, avez-vous eu un résultat de test positif à la COVID-19? 》 (Trois choix de réponse possibles : Oui, Non et Pas de résultat de test, mais je crois que je l'ai contractée [autodiagnostic en fonction de mes symptômes des sept derniers jours])

La question cible permettant de calculer le nombre de personnes ayant eu la COVID-19 sur les sept derniers jours en utilisant l'échantillonnage indirect et la méthode APR est la suivante :

«Combien connaissez-vous de personnes ayant eu un résultat de test positif pour la COVID-19 au cours des sept derniers jours (incluant aujourd'hui)? » 6

Les questions de contrôle utilisées pour obtenir les DRA visent le nombre de connaissances qui sont médecins, qui résident dans une résidence pour personnes âgées (en résidence pour personnes âgées [RPA], en CHSLD ou en ressource intermédiaire ou familiale [RI-RTF]) ou qui ne sont pas vaccinées. Nous disposons d'une estimation précise du nombre total de personnes dans chacune de ces situations au Québec. Le nombre de médecins inscrits au Collège des médecins du Québec (actifs et inactifs) est de 25222 au 17 janvier $2022^{7}$. Selon les estimations les plus récentes disponibles, le nombre total de

6. Un préambule spécifie de considérer tout type de connaissances (famille, amis, collègues de travail, simples connaissances), au Québec.

7. Site du CMQ : http://www.cmq.org/ statistiques/repartition-generale.aspx. 
personnes résidant en RPA, ${ }^{8} \mathrm{CHSLD}^{9}$ ou RI$\mathrm{RTF}^{10}$ est de 181 655. Le nombre d'adultes non vaccinés en date du 13 janvier 2022 était de $515771 .{ }^{11}$ Les écart-types et intervalles de confiance sont calculés par boostrap en tirant 1000 échantillons avec remplacement.

\section{Résultats}

Dans le Tableau 1, nous présentons les estimations du nombre de nouveaux cas basées sur l'échantillonnage direct (incidence chez les adultes), ainsi que sur les deux estimateurs utilisant l'APR (incidence sur la population totale).

Le nombre de personnes ayant la COVID-19 sur la base de tests PCR, de tests rapides et par auto-diagnostic sur les sept derniers jours est de 407430 (58 204/jour). ${ }^{12}$ En excluant les auto-diagnostics, nous obtenons plutôt un estimé de 261863 cas dans les sept derniers jours, ou 37409 cas en moyenne par jour. Par la méthode de l'amplificateur par réseau, nous obtenons des estimations concordantes de 231967 cas pour la semaine (33 138/jour) en utilisant l'estimateur de Habecker et al. (2015) et de

\footnotetext{
8. Données de la SCHL : https:// www.cmhc-schl.gc.ca/fr/professionals/ housing-markets-data-and-research/

11. Compilation à partir des données de l'INSPQ (tableau 2.1) : https://www.inspq.qc.ca/covid-19/ donnees/vaccination.

12. En incluant les auto-diagnostics, nous obtenons que près de $85 \%$ des répondants déclarant avoir été positifs ont rapporté au moins un symptôme.
}

251706 cas (35 958/jour) avec l'estimateur de Killworth et al. (1998). Il est intéressant de noter que malgré des estimations par APR similaires à celle obtenue avec la question directe, l'écart-type est beaucoup plus faible avec la méthode APR puisque celle-ci exploite les réseaux, ce qui augmente la taille effective de la population considérée.

Comme le démontre la Figure 2, ces chiffres sont beaucoup plus élevés que le chiffre officiel publié par l'INSPQ sur la même période, soit 48815 (6973/jour). ${ }^{13}$ Peu importe la méthode et l'estimateur utilisé (en excluant les auto-diagnostics), nous obtenons une incidence environ cinq fois plus élevée que le chiffre officiel. ${ }^{14}$

\section{Conclusions}

Outre les limites inhérentes à toute modélisation statistique énoncées dans la section méthodes et les limites associées aux enquêtes, cette étude exploratoire propose une estimation rétrospective du nombre de cas récents de COVID19. Réalisée à courte échéance, devant la montée des cas et pour combler l'absence d'estimation du nombre de cas au Québec, cette étude montre que les résultats officiels de tests PCR sous-estiment d'un multiple de cinq le nombre de résultats positifs au Québec sur la période.

13. Nous obtenons ce chiffre en pondérant la somme des cas sur les sept derniers jours pour chacune des journées d'enquête (du 13 au 18 janvier 2022) par la proportion des répondants ayant répondu à l'enquête chaque jour.

14. Les nombres de cas que nous obtenons par la méthode directe ne sont que parmi les adultes, alors que les chiffres de l'INSPQ incluent aussi les cas chez les moins de 18 ans. Ces derniers ne représentent toutefois que moins de $15 \%$ des tests PCR réalisés dans les 14 derniers jours selon l'INSPQ. Néanmoins, ce facteur de cinq reste probablement une limite inférieure. 


\begin{tabular}{rrrrr}
\hline & Nb cas $(7 \mathrm{j})$ & écart-type $(7 \mathrm{j})$ & Nb cas $(\mathrm{j})$ & écart-type $(\mathrm{j})$ \\
\hline Direct positifs & 261863 & 24431 & 37409 & 3490 \\
Avec auto-diag. & 407430 & 30925 & 58204 & 4418 \\
APR, Killworth et al. (1998) & 251706 & 10758 & 35958 & 1537 \\
APR, Habecker et al. (2015) & 231967 & 11072 & 33138 & 1582 \\
\hline
\end{tabular}

TABLEAU 1 - Estimation du nombre de nouveaux cas de COVID-19 selon plusieurs méthodes sur une base de sept jours $(7 \mathrm{j})$ et moyenne par jour (j). La première ligne montre l'incidence chez les adultes, basée sur un test positif (PCR ou rapide), à partir de la question individuelle. La deuxième ligne ajoute ceux rapportant qu'ils ont eu la COVID-19 sur la base d'un auto-diagnostic en fonction des symptômes. La troisième ligne montre l'incidence dans la population totale sur la base de la méthode de l'amplificateur par réseau en utilisant l'estimateur par maximum de vraisemblance de Killworth et al. (2003), alors que la dernière ligne utilise plutôt l'estimateur de Habecker et al. (2015).

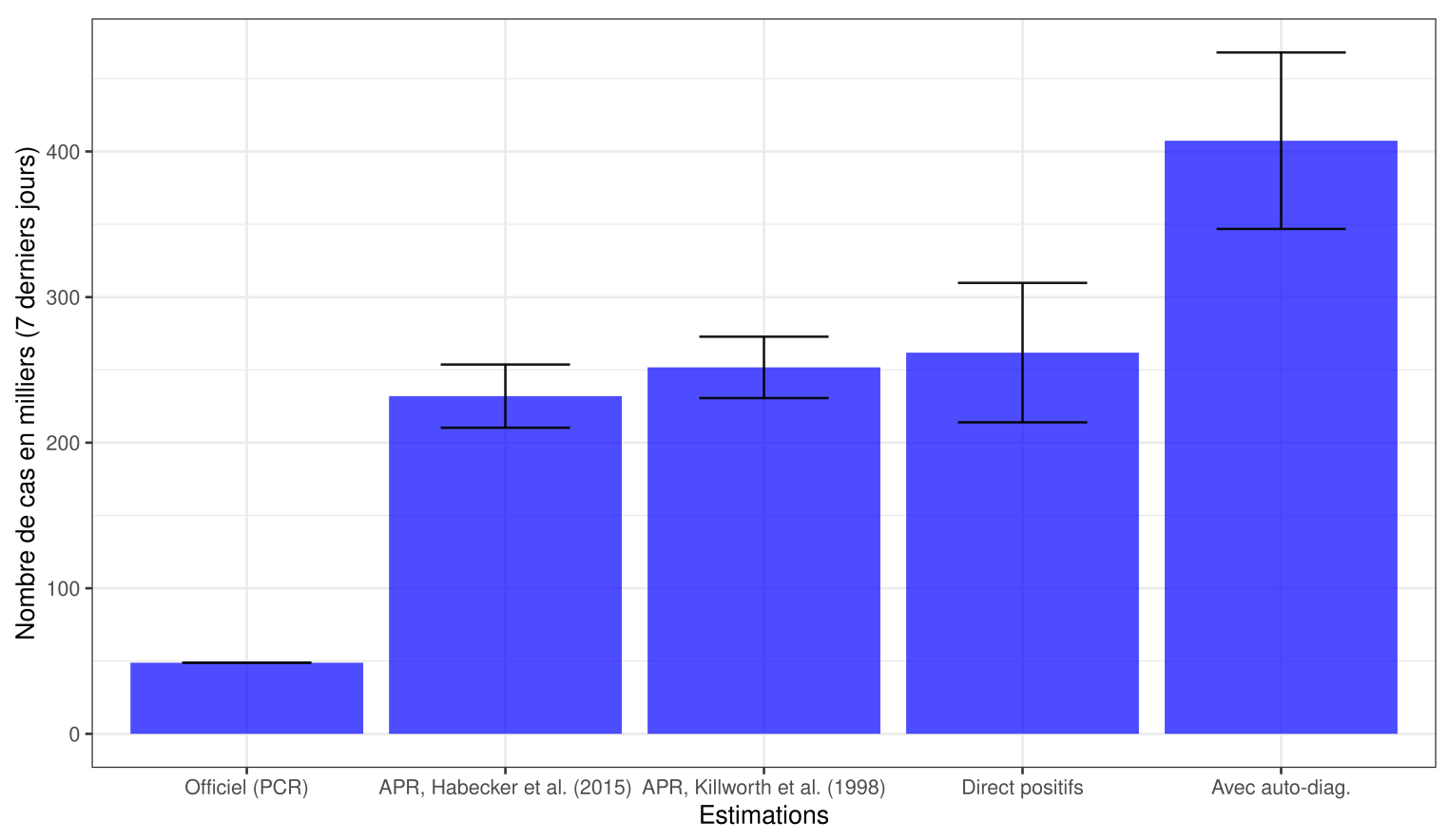

FiguRE 2 - Estimations de l'incidence de la COVID-19, avec intervalles de confiance à 95\%. Nous présentons le nombre de nouveaux cas dans la population totale tel que rapporté par l'INSPQ pour la période du 7 au 13 janvier 2022 (tests PCR positifs); nos estimations selon la méthode de l'amplificateur par réseau (selon les estimateurs de Killworth et al. (1998) et de Habecker et al. (2015)) ; et l'incidence chez les adultes estimée à l'aide de la méthode directe (question individuelle pour les 18 ans et plus). Nous montrons aussi l'incidence chez les adultes en incluant les auto-diagnostics. 
Nos résultats sont cohérents avec ceux de l'Office national des statistiques du Royaume-Uni qui estimait, par échantillonnage représentatif, qu'une personne sur vingt était infectée par la COVID19 au début du mois de janvier. ${ }^{15}$ Sans autre période de référence, il demeure difficile de statuer sur l'évolution de l'incidence des cas. Nous comptons répéter le même exercice dans les quatre prochaines semaines afin d'avoir une meilleure idée de la tendance.

\footnotetext{
15. Données de l'ONS : https://www. ons.gov.uk/peoplepopulationandcommunity/ healthandsocialcare/conditionsanddiseases/ bulletins/coronaviruscovid19infectionsurveypilot/ 5january2022.
}

\section{Références}

Bernard, R. H., Johnsen, E. C., Killworth, P. D., and Robinson, S. (1991). Estimating the size of an average personal network and of an event subpopulation : Some empirical results. Social Science Research, 20(2) :109-121.

Habecker, P., Dombrowski, K., and Khan, B. (2015). Improving the network scale-up estimator : Incorporating means of sums, recursive back estimation, and sampling weights. PLOS ONE, 10 :e0143406.

Killworth, P. D., Johnsen, E. C., McCarty, C., Bernard, H. R., and Shelley, G. A. (2003). Attempting to quantify transmission and barrier errors in scale-up methods.

Killworth, P. D., McCarty, C., Bernard, H. R., Shelley, G. A., and Johnsen, E. C. (1998). Estimation of seroprevalence, rape, and homelessness in the United States using a social network approach. Evaluation review, 22(2) :289307.

Laga, I., Bao, L., and Niu, X. (2021). Thirty Years of The Network Scale-up Method. Journal of the American Statistical Association, 116(535) :1548-1559.

Rolland-Guillard, L., Charrance, G., and Morand, E. (2020). Quelles méthodes pour estimer la taille d'une population difficile à enquêter? 


\section{ANNEXE : QUESTIONNAIRE DE L'ENQUÊTE}


Vous trouverez ci-après un questionnaire anonyme auquel nous vous invitons à répondre. Ce questionnaire a été développé dans le cadre d'un projet de recherche conjoint de HEC Montréal et du Centre Interuniversitaire de Recherche en Analyse des Organisations (CIRANO).

Il n'y a pas de limite de temps pour répondre au questionnaire, bien que nous ayons estimé que cela devrait vous prendre environ 5 minutes.

Les renseignements recueillis sont anonymes et resteront strictement confidentiels; ils ne seront utilisés que pour l'avancement des connaissances et la diffusion des résultats globaux dans des forums savants ou professionnels. Il se peut que les données recueillies soient partagées avec d'autres chercheurs à des fins de recherche non-commerciale uniquement et, possiblement, dans le cadre de projets autres que celui pour lesquelles les données auront été recueillies à l'origine. La base de données anonymisée résultant de l'enquête pourrait être ultérieurement rendue disponible publiquement à des fins de recherche académique.

Le fournisseur de collecte de données s'engage à ne révéler aucune information personnelle (ou toute autre information relative aux participants de cette étude) à d'autres utilisateurs ou à tout autre tiers, à moins que le répondant consente expressément à une telle divulgation ou que celle-ci soit exigée par la loi.

Vous êtes complètement libre de refuser de participer à ce projet et vous pouvez décider en tout temps d'arrêter de répondre aux questions.

Le fait de remplir ce questionnaire sera considéré comme votre consentement à participer à notre recherche et à l'utilisation des données recueillies dans ce questionnaire pour d'éventuelles recherches futures.

Puisque le questionnaire est anonyme, une fois votre participation complétée, il vous sera impossible de vous retirer du projet de recherche, car il sera impossible de déterminer quelles réponses sont les vôtres.

Si vous avez des questions concernant cette recherche, vous pouvez contacter le chercheur principal, Pierre-Carl Michaud, au numéro de téléphone ou à l'adresse courriel indiqués ci-dessous.

Le comité d'éthique de la recherche de HEC Montréal a statué que la collecte de données liée à la présente étude satisfait aux normes éthiques en recherche auprès des êtres humains. Pour toute question en matière d'éthique, vous pouvez communiquer avec le secrétariat de ce comité par courriel à cer@hec.ca.

Merci de votre précieuse collaboration!

Pierre-Carl Michaud

Professeur titulaire

Département d'économie appliquée

HEC Montréal

514-340-6466

pierre-carl.michaud@hec.ca

En cliquant sur le bouton « Continuez », vous consentez à participer à la recherche. 
INSTRUCTIONS GÉNÉRALES DE PROGRAMMATION :

[TYPE DE PROJET : Web]

[LANGUES : FR/EN]

[TRACKING : Oui]

NOTE AU LECTEUR : Texte en vert et en gris = consignes de programmation

[POSER POSTAL3 À TOUS] / [OUVERTE : VALIDATION - FORCER LE FORMAT A9A]

POSTAL3

Veuillez indiquer les 3 premiers caractères de votre code postal.

(INSCRIRE LE CODE POSTAL EN FORMAT A9A)

\begin{tabular}{|l|c|c|c|}
\hline Label & Value & Attribute & Termination \\
\hline Je préfère ne pas répondre & A9A & & \\
\hline
\end{tabular}

[POSER QOQC SI PROV=QC] / [MENTION SIMPLE]

Q0QC. Dans quelle région du Québec demeurez-vous?

\begin{tabular}{|l|c|c|c|}
\hline Libellé & Valeur & Attribut & Terminaison \\
\hline Bas-Saint-Laurent & 1 & & \\
\hline Saguenay-Lac-Saint-Jean & 2 & & \\
\hline Capitale-Nationale & 3 & \\
\hline Mauricie & 4 & \\
\hline Estrie & 5 & \\
\hline Montréal & 6 & \\
\hline Outaouais & 7 & \\
\hline Abitibi-Témiscamingue & 8 & \\
\hline Côte-Nord & 9 & \\
\hline Nord-du-Québec & 10 & \\
\hline Gaspésie/lles-de-la-Madeleine & 11 & \\
\hline Chaudière-Appalaches & 12 & & \\
\hline Laval & 13 & & \\
\hline Lanaudière & 14 & & \\
\hline Laurentides & 15 & & \\
\hline Montérégie & 16 & & \\
\hline Centre-du-Québec & 17 & & \\
\hline
\end{tabular}

À Q0QCG

Une série de questions est ensuite insérée ici pour identifier la provenance plus précise des répondants.

[CALCULÉE - POSER REGIO SI PROV=QC]

REGIO

\begin{tabular}{|l|c|c|c|}
\hline Libellé & Valeur & Attribut & Terminaison \\
\hline MTL RMR & 1 & & \\
\hline QC RMR & 2 & & \\
\hline AUTRES RÉGIONS & 3 & & \\
\hline
\end{tabular}


[POSER À TOUS]

SEXE.

Êtes-vous de sexe :

Note : comme indiqué par Statistique Canada, les Canadiens transgenres, transsexuels et intersexués doivent indiquer le sexe (masculin ou féminin) auquel ils s'identifient le plus.

$\square$ Masculin (1)

$\square$ Féminin (2)

[POSER À TOUS] / [MENTION SIMPLE]

AGE. Quel âge avez-vous ?

\begin{tabular}{|l|c|c|c|}
\hline Libellé & Valeur & Attribut & Terminaison \\
\hline Moins de 18 ans & 0 & & TERMINER \\
\hline De 18 à 24 ans & 1 & & \\
\hline De 25 à 29 ans & 2 & & \\
\hline De 30 à 34 ans & 3 & & \\
\hline De 35 à 39 ans & 4 & & \\
\hline De 40 à 44 ans & 5 & & \\
\hline De 45 à 49 ans & 6 & & \\
\hline De 50 à 54 ans & 7 & & \\
\hline De 55 à 59 ans & 8 & & \\
\hline De 60 à 64 ans & 9 & & \\
\hline De 65 à 69 ans & 10 & & \\
\hline De 70 à 74 ans & 11 & & \\
\hline 75 ans ou plus & 12 & & TERMINER \\
\hline Je préfère ne pas répondre & 9 & & \\
\hline
\end{tabular}

[POSER À TOUS]

[MENTION SIMPLE]

LANGU. Quelle est la langue que vous avez apprise en premier lieu à la maison dans votre enfance et que vous comprenez toujours?

\begin{tabular}{|l|c|c|c|}
\hline Libellé & Valeur & Attribut & Terminaison \\
\hline Français & 1 & & \\
\hline Anglais & 2 & & \\
\hline Autre & 3 & \\
\hline Français et anglais & 7 & \\
\hline Français et autres & 4 & \\
\hline Anglais et autres & 5 & & \\
\hline Autres et autres & 6 & & \\
\hline Je préfère ne pas répondre & 9 & & \\
\hline
\end{tabular}


POSER FOY1 À TOUS]

[NUMÉRIQUE : Max=20] / [DÉCIMALE : 0] / [TYPE DE SYMBOLE : personne(s)] / [EMPLACEMENT DU

SYMBOLE : Après]

FOY1. En vous incluant, combien de personnes composent votre ménage en incluant les adultes et les enfants?

personne(s)

\begin{tabular}{|l|c|c|c|}
\hline Libellé & Valeur & Attribut & Terminaison \\
\hline Une seule (moi-même) & 1 & & \\
\hline Je préfère ne pas répondre & 99 & \\
\hline
\end{tabular}

[POSER FOY2 SI FOY1 > 1 ET FOY1 < 99]

[NUMÉRIQUE : BORNES Min=1, Max=20] / [DÉCIMALE : 0] / [TYPE DE SYMBOLE : enfant(s)] /

[EMPLACEMENT DU SYMBOLE : Après]

FOY2. Parmi ces personnes, combien d'enfants des groupes d'âge suivants habitent avec vous (la moitié du temps ou plus)?

0 à 4 ans : Numérique entier $[0,+)$

5 à 12 ans : Numérique entier $[0,+)$

13 à 17 ans : Numérique entier $[0,+)$

0 valeur par défaut dans chaque cellule d'âge, la somme de 1,2,3 ne devrait pas dépasser [\#Q11]-1

[POSER À TOUS]

[MENTION SIMPLE]

SCOL. À quel niveau se situe la dernière année de scolarité que vous avez terminée ?

\begin{tabular}{|l|c|c|}
\hline Libellé & Valeur & Attribut \\
\hline Primaire (7 ans ou moins) & 1 & \\
\hline Secondaire (formation générale ou professionnelle (8 à 12 ans)) & 2 & \\
\hline $\begin{array}{l}\text { Collégial (formation préuniversitaire, formation technique, certificats, } \\
\text { attestations ou diplômes de perfectionnement) }\end{array}$ & 3 \\
\hline Universitaire certificats et diplômes & 4 \\
\hline Universitaire 1er cycle Baccalauréat (incluant cours classique) & 5 & \\
\hline Universitaire 2e cycle Maîtrise & 6 & \\
\hline Universitaire 3e cycle Doctorat & 7 & \\
\hline Je préfère ne pas répondre & 9 & \\
\hline
\end{tabular}

INSÉRER FILT1 
[POSER À TOUS] / [MENTIONS MULTIPLES (4 max)]

[Le choix 97 ne peut être combiné avec d'autres choix, pour les autres, choix multiple possible]

Q1 Dans les 7 derniers jours, avez-vous ressenti des symptômes que l'on pourrait associer à la COVID-19? En vous basant sur la liste suivante présentée par le Gouvernement du Québec, veuillez sélectionner chacun des groupes de symptômes ressentis.

Considérez les 7 derniers jours, en comptant aujourd'hui.

\begin{tabular}{|c|c|}
\hline $\begin{array}{l}\text { Fièvre } \\
\left(\text { plus de } 38^{\circ} \mathrm{C}\right)\end{array}$ & 1 \\
\hline $\begin{array}{l}\text { Symptômes généraux } \\
\text { (perte soudaine d'odorat sans congestion nasale, perte de goût, grande } \\
\text { fatigue, perte d'appétit importante, douleurs musculaires généralisées -non } \\
\text { liées à un effort physique-, mal de tête, sueurs nocturnes) }\end{array}$ & 2 \\
\hline $\begin{array}{l}\text { Symptômes respiratoires } \\
\text { (toux nouvelle ou aggravée, essoufflement, difficulté à respirer, mal de } \\
\text { gorge, nez qui coule ou congestion nasale de cause inconnue) }\end{array}$ & 3 \\
\hline $\begin{array}{l}\text { Symptômes gastro-intestinaux } \\
\text { (nausées, vomissements, diarrhée, maux de ventre) }\end{array}$ & 4 \\
\hline Aucun symptôme & 97 \\
\hline
\end{tabular}

\section{[POSER À TOUS] / [MENTION SIMPLE]}

Q2 Avez-vous été en isolement dû à la COVID-19 dans les 7 derniers jours, incluant aujourd'hui?

$\begin{array}{ll}1 & \text { Oui } \\ 2 & \text { Non }\end{array}$

[POSER À TOUS] / [MENTION SIMPLE]

Q3 Dans les 7 derniers jours incluant aujourd'hui, avez-vous eu un résultat de test positif à la COVID-19?

$1 \quad$ Oui

2 Non

3 Pas de résultat de test, mais je crois que je l'ai contractée (autodiagnostic en fonction de mes symptômes des 7 derniers jours)

[POSER Si Q3=1] / [MENTION SIMPLE]

Q3a Par quel type de test avez-vous reçu ce résultat positif?

$1 \quad$ Test PCR en centre de dépistage

2 Test rapide

3 Les deux (par exemple, un test rapide suivi d'un test PCR)

[POSER Si Q3=2] / [MENTION SIMPLE]

Q3b Selon vous, sur une échelle de 0 à 100, quelles sont les chances que vous développiez des symptômes associés à la COVID-19 dans les 7 prochains jours? 
Indiquez 0 si vous n'avez aucune chance de développer des symptômes et 100 s'il est certain que vous allez développer des symptômes.

Numérique $[0,100]$.

Permettre : 999 Ne sait pas.

INSÉRER QFIL2

[AFFICHER À TOUS] / [MENTION SIMPLE]

Les prochaines questions concernent votre réseau de connaissances personnelles au Québec, c'est à dire les gens que vous connaissez, et qui vous connaissent, personnellement (exemple: famille, amis, collègues de travail, simples connaissances, etc.)

(Répétez cette phrase sur l'écran pour chaque question Q4 à Q8)

[POSER À TOUS] / [MENTION SIMPLE]

Q4 Combien connaissez-vous de personnes ayant eu un résultat de test positif pour la COVID-19 au cours des 7 derniers jours (incluant aujourd'hui)?

Numérique entier $[0,999)$

[POSER À TOUS] / [MENTION SIMPLE]

Q5 Combien connaissez-vous de personnes qui ont été en isolement dû à la COVID-19 au cours des 7 derniers jours (incluant aujourd'hui)?

Numérique entier $[0,+)$

[POSER À TOUS] / [MENTION SIMPLE]

Q6 Combien connaissez-vous de personnes qui sont médecin?

Numérique entier $[0,+)$

[POSER À TOUS] / [MENTION SIMPLE]

Q7 Combien connaissez-vous de personnes qui résident dans une résidence pour personne âgée (RPA), en ressource intermédiaire ou familiale (RI-RTF) ou en CHSLD?

Numérique entier $[0,+)$

[POSER À TOUS] / [MENTION SIMPLE]

Q8 Combien connaissez-vous de personnes (de 18 ans ou plus) n'ayant reçu aucune dose de vaccin contre la COVID-19?

Numérique entier $[0,+)$ 
[POSER À TOUS] / [MENTION SIMPLE]

Q9 Au moment de cette enquête comment pourriez-vous qualifier votre statut vaccinal pour la COVID-19?

$1 \quad$ Vacciné(e) 3 doses

2 Vacciné(e) 2 doses

3 Vacciné(e) 1 dose

4 Aucune dose reçue

99 Je préfère ne pas répondre

\section{INSÉRER FILT3}

[AFFICHER À TOUS]

M1. C'est presque terminé. Les prochaines questions serviront à des fins statistiques seulement.

[POSER À TOUS] / [MENTIONS MULTIPLES ]

EMPLO. Quelle est votre situation d'emploi actuelle?

\begin{tabular}{|l|c|l|l|}
\hline Libellé & Valeur & Attribut & Terminaison \\
\hline Employé à temps plein & 1 & & \\
\hline Employé à temps partiel & 2 & \\
\hline À votre compte / travailleur autonome & 3 & \\
\hline Étudiant & 4 & \\
\hline Au foyer & 5 & \\
\hline Sans emploi & 6 & \\
\hline Retraité & 7 & \\
\hline Je préfère ne pas répondre & 9 & \\
\hline
\end{tabular}

[POSER SI EMPLO=1,2,3] / [MENTION SIMPLE]

Q10 Quelle affirmation décrit le mieux votre situation au travail cette semaine?

1 Au travail en personne exclusivement

2 En télétravail exclusivement

3 Au travail en personne et en télétravail

4 Absent (ex : vacances, maladie, isolement)

[POSER SI EMPLO=1,2,3] / [MENTION SIMPLE]

Q11 Laquelle des industries suivantes décrit le mieux l'endroit où vous occupez un emploi, que vous soyez absent ou non du travail?

1 Agriculture, foresterie, pêche et chasse

2 Extraction minière, exploitation en carrière, et extraction de pétrole et de gaz

3 Services publics

4 Construction

5 Fabrication 
6 Commerce de gros

7 Commerce de détail

8 Transport et entreposage

9 Industrie de l'information et industrie culturelle

10 Finance et assurances

11 Services immobiliers et services de location et de location à bail

12 Services professionnels, scientifiques et techniques

13 Gestion de sociétés et d'entreprises

14 Services administratifs, services de soutien, services de gestion des déchets et services d'assainissement

15 Services d'enseignement

16 Soins de santé et assistance sociale

17 Arts, spectacles et loisirs

18 Services d'hébergement et de restauration

19 Autres services (sauf les administrations publiques)

20 Administrations publiques

[POSER MARIT À TOUS] / [MENTION SIMPLE]

MARIT. Êtes-vous ...?

\begin{tabular}{|c|c|c|c|}
\hline Libellé & Valeur & Attribut & Terminaison \\
\hline Célibataire & 1 & & \\
\hline Marié(e) ou conjoint(e) de fait & 2 & & \\
\hline Veuf (veuve) & 3 & & \\
\hline Séparé(e) & 4 & & \\
\hline Divorcé(e) & 5 & & \\
\hline Je préfère ne pas répondre & 9 & & \\
\hline
\end{tabular}

[POSER REVEN À TOUS] / [MENTION SIMPLE]

REVEN. Parmi les catégories suivantes, laquelle reflète le mieux le REVENU total avant impôt de tous les membres de votre foyer pour l'année 2021 ?

\begin{tabular}{|l|c|c|c|}
\hline Libellé & Valeur & Attribut & Terminaison \\
\hline $19999 \$$ et moins & 1 & & \\
\hline De $20000 \$$ à $39999 \$$ & 2 & \\
\hline De $40000 \$$ à $59999 \$$ & 3 & \\
\hline De $60000 \$$ à $79999 \$$ & 4 & \\
\hline De $80000 \$$ à $99999 \$$ & 5 & \\
\hline $100000 \$$ et plus & 6 & \\
\hline Je préfère ne pas répondre & 9 & \\
\hline
\end{tabular}

